\title{
Detecting Acoustic Blackbody Radiation
}

\section{A nanomechanical resonator detects and isolates acoustic waves from a remote thermal source.}

\section{By Christopher Crockett}

A s objects heat up, they not only glow but also emit acoustic energy. This "acoustic blackbody radiation" may be as ubiquitous as its more famous electromagnetic cousin but is typically faint and difficult to characterize. Now, a pair of researchers have used a nanomechanical resonator to detect acoustic blackbody radiation from a remote source [1]. They say their technique-the acoustic analog of remote infrared thermometry-could lead to improvements in applications ranging from metrology to quantum information.

Thomas Purdy, of the University of Pittsburgh, and Robinjeet Singh, of the University of Maryland, College Park, suspended a $1-\mathrm{mm}^{2}$ membrane of silicon nitride in a window cut within a silicon substrate. On the substrate, several millimeters from the membrane, they deposited four oblong blobs of epoxy composite that acted as acoustic blackbodies. When heated by a laser, the blackbodies emitted acoustic energy, which traveled through the substrate and excited particular patterns of vibrations in the membrane. The researchers detected these

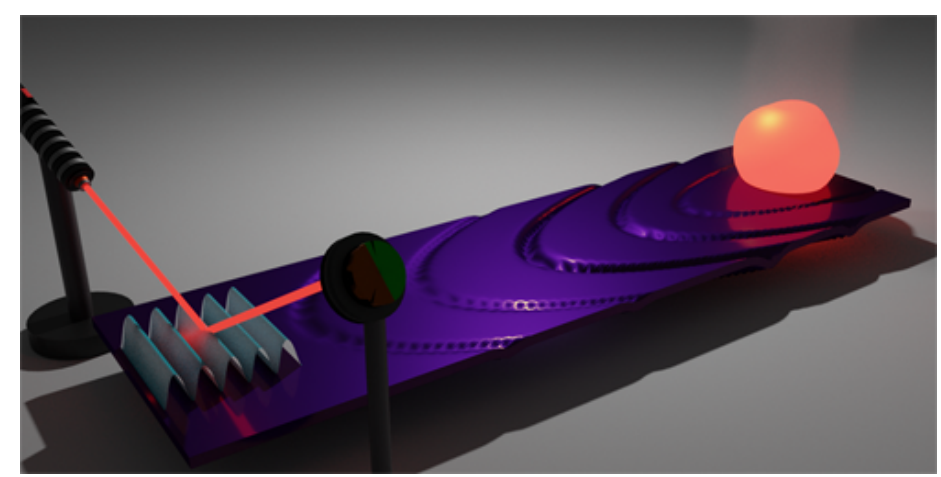

Credit: R. Singh/University of Maryland, College Park vibrations with a second laser.

By varying the power of the heating laser, the duo found that the acoustic energy in the resonator directly tracked the temperature of the blackbody, and the vibration patterns were different depending on which blackbody was being heated. They estimate that $99 \%$ of the thermal motion in the resonator came from the blackbodies, thus showing significant immunity to "self-heating" from the probe laser-an effect that plagues conventional optomechanical thermometers. The researchers hope to leverage this setup to create an accurate, compact device that can be used outside the lab.

Christopher Crockett is a freelance writer based in Arlington, Virginia.

\section{REFERENCES}

1. R. Singh and T. P. Purdy, "Detecting acoustic blackbody radiation with an optomechanical antenna," Phys. Rev. Lett. 125, 120603 (2020). 